\title{
Cardiogenic Shock due to COVID-19-Related Myocarditis in a 19-Year-Old Autistic Patient
}

\author{
Greta Pascariello ${ }^{\mathrm{a}, \mathrm{c}}$, Giuliana Cimino ${ }^{\mathrm{a}}$, Emiliano Calvi ${ }^{\mathrm{a}}$, Nicola Bernardi ${ }^{\mathrm{a}}$, Michele Grigolato ${ }^{\mathrm{b}}$, \\ Polyxeni Garyfallidis ${ }^{\mathrm{b}}$, Davide Fabbricatore ${ }^{\mathrm{a}}$, Elisa Pezzola ${ }^{\mathrm{a}}$, Carlo M. Lombardia \\ Marco Metra ${ }^{a}$, Enrico Vizzardia
}

\begin{abstract}
Severe acute respiratory syndrome coronavirus 2 (SARS-CoV-2) is mainly responsible for respiratory involvement but cardiac complications are also reported. Nevertheless, potential life-threatening conditions in young people have not been described. A 19-year-old male autistic patient was admitted with fever and cough. The chest radiography showed viral pneumonia and the nasopharyngeal swab detected SARS-CoV-2. He rapidly developed hypotension, oliguria and increased myocardial injury markers and was treated with adrenaline, antiviral drugs and mechanical ventilation. Echocardiography revealed diffuse myocardial hypo-akinesia and decreased left ventricular ejection fraction (LVEF). After several days of treatment, the patient was weaned off mechanical ventilation, LVEF recovered to $50 \%$ and laboratory tests showed a decrease of markers of myocardial injury. Coronavirus disease 2019 (COVID-19) can therefore severely affect myocardium with life-threatening complications and even young people can be involved.
\end{abstract}

Keywords: COVID-19; Myocarditis; Cardiogenic shock; Echocardiography

\section{Introduction}

Severe acute respiratory syndrome coronavirus 2 (SARS$\mathrm{CoV}-2)$, responsible for the novel virus named coronavirus disease 2019 (COVID-19), first appeared in Wuhan, China, on December 2019 [1] and then rapidly spread worldwide reaching pandemic levels on March 2020 [2].

Although respiratory involvement, ranging from mild

Manuscript submitted June 1, 2020, accepted June 9, 2020

Published online June 29, 2020

aDepartment of Medical and Surgical Specialties, Institute of Cardiology, University of Brescia, Italy

${ }^{b}$ Department of Policardiografia, Spedali Civili, Brescia, Italy

${ }^{\mathrm{c} C}$ Corresponding Author: Pascariello Greta, Department of Medical and Surgical Specialties, Institute of Cardiology, University of Brescia, Italy. Email: greta.pascariello@hotmail.it

doi: https://doi.org/10.14740/jmc3517 flu-like illness to potentially fatal acute respiratory distress syndrome (ARDS), is the dominant clinical manifestation of COVID-19, severe and acute cardiovascular complications are described, including myocardial injury and myocarditis, acute myocardial infarction (AMI), heart failure, dysrhythmias and venous thromboembolic events (VTEs) [3, 4].

Among the general population, children are less frequently affected. By March, in Italy, children younger than 18 years of age who had COVID-19 were only $1 \%$ of the total patients [5]. Childhood cases of COVID-19 are more likely asymptomatic or with mild respiratory or gastrointestinal symptoms [6].

Herein we describe a case of cardiogenic shock due to COVID-19-related myocarditis in a young Italian patient.

\section{Case Report}

A 19-year-old Italian male patient, suffering from autistic spectrum disorder, visited our emergency department in April 2020 because of a worsening of the flu syndrome, and showed up with fever, cough, accompanied by diarrhea and vomit since 3 days. In the previous days, at home, he received treatment with amoxicillin/clavulanic acid without clinical improvement.

On arrival to our attention, physical examination revealed blood pressure of 110/70 $\mathrm{mm} \mathrm{Hg}$, heart rate of 140 beats per minute, body temperature of $40{ }^{\circ} \mathrm{C}$ and oxygen saturation of $90 \%$, so non-invasive ventilation was initiated. Blood samples showed high inflammatory markers with raised white blood cell count (WBC, $\left.15.59 \times 10^{3} / \mu \mathrm{L}\right)$, neutrophilia $\left(14.64 \times 10^{3} /\right.$ $\mu \mathrm{L})$, lymphopenia $\left(0.51 \times 10^{3} / \mu \mathrm{L}\right)$ and increased C-reactive protein values $(401 \mathrm{mg} / \mathrm{L})$ (Table 1). Arterial blood gas analysis revealed a $\mathrm{pH}$ of 7.51, $\mathrm{PCO}_{2}$ of $26 \mathrm{~mm} \mathrm{Hg}, \mathrm{PO}_{2}$ of $103 \mathrm{~mm}$ $\mathrm{Hg}$ and $\mathrm{SaO}_{2}$ at $99.8 \%$ while on oxygen therapy with non-rebreather mask. Bilateral interstitial pneumonia with thickening and interstitial infiltrates suggestive of viral pneumonia was observed in chest X-ray and then samples of swab, taken from his upper respiratory tract, detected SARS-COV-2 through real-time reverse transcriptase-polymerase chain reaction assay (real-time PCR). The patient was then admitted to infectious disease ward with a treatment regime that consisted of oxygen therapy at $10 \mathrm{~L} / \mathrm{min}$ with non-rebreather mask, hydroxychloroquine and ceftriaxone (patient allergic to macrolides).

The day after the admission, because of a sudden clinical deterioration due to cardiogenic shock with severe hypoten- 


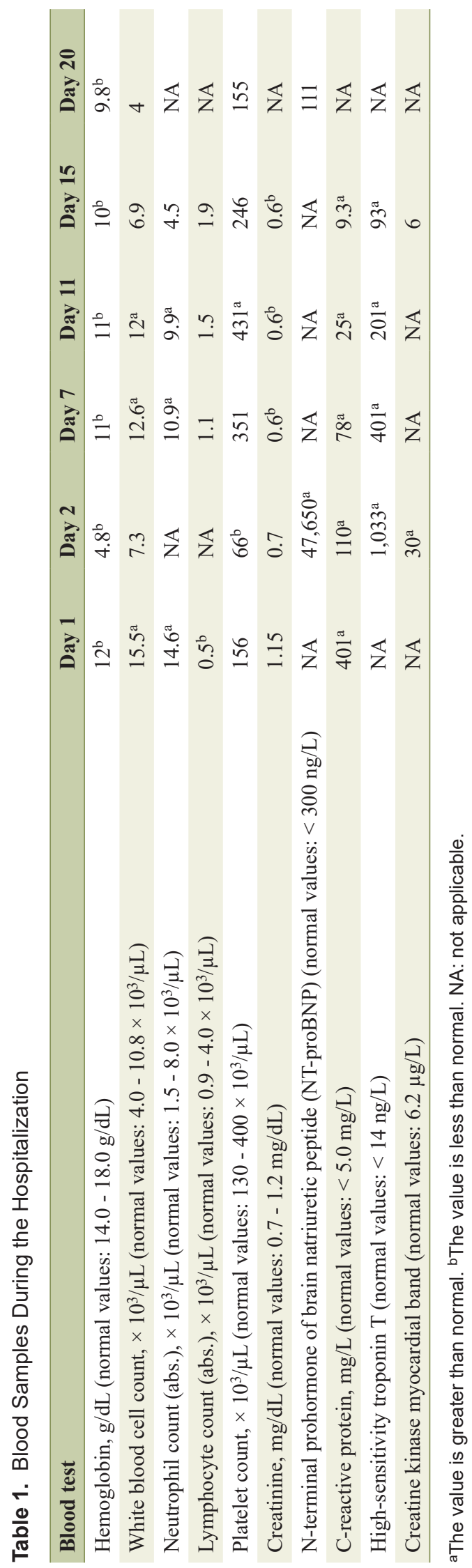

sion, tachycardia, oliguria, anemia and marked desaturation, the patient was immediately transferred to intensive care unit (ICU) where he was treated with adrenaline, high-flow oxygen (HFN), later replaced by orotracheal intubation because of worsening of gas exchanges, hemotransfusion, levofloxacin (500 mg twice daily), antiviral therapy with remdesivir (100 $\mathrm{mg}$ once daily) and oseltamivir (150 mg once daily), anticoagulation therapy with low molecular weight heparin (LMWH) (6,000 IU twice daily), diuretic, pantoprazole (20 mg twice daily) and dexamethasone (10 $\mathrm{mg}$ once daily). The domiciliary therapy with aripiprazole (20 mg once daily) was regularly fed. Bedside echocardiography showed an enlarged left ventricle $(56 \mathrm{~mm})$, diffuse myocardial hypo-akinesia along with severe left ventricular ejection systolic dysfunction (LVEF, 15$20 \%$ ) and decrease in right cardiac function. The chest computed tomography (CT) ruled out acute pulmonary embolism and revealed cardiomegaly. Blood test showed elevation of myocardial injury markers such as high-sensitivity troponin $\mathrm{T}$ $(1,033 \mathrm{ng} / \mathrm{L})$, creatine kinase myocardial band $(30 \mu \mathrm{g} / \mathrm{L})$ and $\mathrm{N}$-terminal pro-brain natriuretic peptide (NT-proBNP) $(47,650$ $\mathrm{ng} / \mathrm{L}$ ), so that a diagnosis of acute myocarditis was formulated. Additional tests for common cardiotropic agents were negative, so we evinced that COVID-19 was the leading cause of our patient's myocarditis complicated by cardiogenic shock.

After a few days, the patient was weaned off mechanical ventilation, and adrenalina was replaced by dobutamine. He was discharged from ICU after 2 weeks and transferred to cardiologic unit where the patient's clinical conditions gradually improved. Chest radiography showed improvement of the radiologic findings, LVEF recovered to $50 \%$ without kinetic disorders and two-dimensional global longitudinal strain (2DGLS) was $12 \%$ at the transthoracic echocardiography performed on day 20 and markers of myocyte necrosis progressively stabilized and reduced (Table 1).

During the hospitalization, low doses of ramipril (2.5 mg) and bisoprolol $(2.5 \mathrm{mg})$, for evidence of sinus tachycardia, were started and well tolerated. We decided not to perform cardiovascular magnetic resonance or an endomyocardial biopsy due to the important patient's comorbidity and the poor compliance. After 3 weeks, the patient was discharged, hemodynamically stable and asymptomatic.

\section{Discussion}

Herein we describe a case of acute myocarditis with rapid occurrence of cardiogenic shock. In literature, recently published case reports have shown that COVID-19 can be responsible for myocarditis [7] and even fulminant myocarditis [8]. One study suggested that up to $7 \%$ of COVID-19-related deaths were due to myocarditis [9]. The underlying pathogenetic pathways can be related to either direct myocardial injury due to viral load or the effect of systemic inflammation. High viral loads and mononuclear infiltrates were detected in autopsies of some patients with COVID-19 and myocarditis [3]. Tavazzi et al recently described the first biopsy-proven acute cardiac injury directly linked to myocardial localization of the SARS-CoV-2 rapidly degenerating into cardiogenic shock. Endomyocardial biopsy 
demonstrated low grade myocardial inflammation and viral particles in the myocardium, suggesting either a viremic phase or alternatively infected macrophage migration from the lung. They observed viral particles in interstitial cells of the myocardium, but did not observe viral particles in cardiac myocyte. Cardiac cells showed nonspecific damage mainly represented by myofibrillar lysis [10]. Other studies are so needed to confirm viral cardiotropism.

To our knowledge, in literature there are not reports of cardiogenic shock in a so young population. In contrast with infected adults, children and young people appear to have a milder clinical course as reported by data available from China and United States $[6,11]$. These findings are largely consistent with the Coronavirus Infection in Pediatric Emergency Departments (CONFIDENCE) Study performed by an Italian research group in order to determine the spectrum of disease in children in Italy. CONFIDENCE Study involved a cohort of 100 Italian children younger than 18 years of age with confirmed diagnosis of COVID-19 who were assessed in March in 17 pediatric emergency departments. The median age of the children was 3.3 years, $21 \%$ were asymptomatic, $58 \%$ had mild disease, $19 \%$ had moderate disease, $1 \%$ had severe disease and $1 \%$ were in critical conditions. Severe and critical cases were diagnosed in patients with coexisting illnesses. No deaths were reported [12].

Nowadays extracorporeal membrane oxygenation (ECMO) represents the best choice for the management of cardiogenic shock in adult patients with minor or no comorbidities. Among paediatric population, ECMO is less frequently employed and extracorporeal life support (ECLS), delivered to neonates ( 0 - 28 days) for respiratory support, is the largest subcategory of ECLS among children $<18$ years. This is described by a report that analyzes data from children aged $0-17$ years in the Extracorporeal Life Support Organization (ELSO) registry between 2009 and 2015 [13].

ELSO registry, through guidelines development, research and data management, is the largest available resource to provide support to institutions delivering extracorporeal life assistance. In our young patient, ECMO was not used even due to its potential neurological complications. The case report, in fact, deals with a serious cardiovascular complication in an autistic patient raising the question whether the virus can be more aggressive in neuropsychiatric disorders. To date we know that SARS-CoV-2 is a neurotropic virus that penetrates the central nervous system (CNS) through several routes, most notably through intranasal inoculation and through peripheral nerves using trans-synaptic pathways [14]. Long-term neuropsychiatric disorders following COVID-19 infection are currently not known and only in the next months or years it will be even possible to know them [15]. Potential mechanism of neuropsychiatric manifestation in COVID-19 can be due to the neuroinvasive potential of COVID-19 but also to the peripheral cytokines involved in the host antiviral response that may compromise blood-brain interface integrity [14].

\section{Conclusions}

Currently it is recognized that COVID-19 can be localized in tissues other than the lung, mainly in heart and CNS. We can consider COVID-19 an eclectic virus with different clinical presentations in the myocardium, ranging from mild abnormalities in markers of myocardial injury to cardiogenic shock.

Our case report discusses a potential life-threatening complication of COVID-19 in a young patient without previous cardiac history, highlighting that even young people can be severely affected. Clinicians, in our opinion, should so maintain a high index of suspicion for COVID-19 infection in children and young people, particularly among those with underlying comorbidities.

Additional studies are required to better understand the exact cardiotropic relevance of the new SARS-CoV-2 virus and the potential aggressiveness in patients with neuropsychiatric disorders.

\section{Acknowledgments}

None to declare.

\section{Financial Disclosure}

None to declare.

\section{Conflict of Interest}

None to declare.

\section{Informed Consent}

The manuscript has been successfully de-identified to protect the patient.

\section{Author Contributions}

All authors contributed to the editing of the manuscript.

\section{Data Availability}

The authors declare that data supporting the findings of this study are available within the article.

\section{Abbreviations}

SARS-CoV-2: severe acute respiratory syndrome coronavirus 2; LVEF: left ventricular ejection fraction; COVID-19: coronavirus disease 2019; AMI: acute myocardial infarction; VTE: venous thromboembolic event; WBC: white blood cell count; $\mathrm{PCO}_{2}$ : partial pressure of carbon dioxide; $\mathrm{PO}_{2}$ : partial pressure of oxygen; $\mathrm{SaO}_{2}$ : oxygen saturation; RT-PCR: real-time reverse transcriptase-polymerase chain reaction assay; ICU: 
intensive care unit; HFN: high-flow oxygen; LMWH: low molecular weight heparin; CT: computed tomography; NT-proBNP: N-terminal pro-brain natriuretic peptide; 2DGLS: twodimensional global longitudinal strain; ECMO: extracorporeal membrane oxygenation; ELSO: Extracorporeal Life Support Organization; CNS: central nervous system

\section{References}

1. World Health Organization. Novel coronavirus - China. Accessed on January, 2020. https:/www.who.int/csr/ don/12-january-2020-novel-coronavirus-china/en.

2. World Health Organization. WHO Director-General's opening remarks at the media briefing on COVID-19 -11March2020.Availableonline online at https://www. who.int/dg/speeches/detail/who-director-general-sopening-remarks-at-the-media-briefing-on-covid-19--20-march-2020.

3. Long B, Brady WJ, Koyfman A, Gottlieb M. Cardiovascular complications in COVID-19. Am J Emerg Med. 2020.

4. Bansal M. Cardiovascular disease and COVID-19. Diabetes Metab Syndr. 2020;14(3):247-250.

5. Italian National Health Institute (Istituto Superiore di Sanita). Coronavirus epidemic: situation report. March 26, 2020. (In Italian) https://www.epicentro.iss.it/coronavirus/bollettino/Bollettino-sorveglianza-integrata-COVID-19_26-marzo\%202020.pdf.

6. Hong $\bar{H}$, Wang Y, Chung HT, Chen CJ. Clinical characteristics of novel coronavirus disease 2019 (COVID-19) in newborns, infants and children. Pediatr Neonatol. 2020;61(2):131-132.

7. Inciardi RM, Lupi L, Zaccone G, Italia L, Raffo M, To- masoni D, Cani DS, et al. Cardiac involvement in a patient with coronavirus disease 2019 (COVID-19). JAMA Cardiol. 2020.

8. Zeng JH, Liu YX, Yuan J, Wang FX, Wu WB, Li JX, Wang LF, et al. First case of COVID-19 complicated with fulminant myocarditis: a case report and insights. Infection. 2020.

9. Ruan Q, Yang K, Wang W, Jiang L, Song J. Correction to: Clinical predictors of mortality due to COVID-19 based on an analysis of data of 150 patients from Wuhan, China. Intensive Care Med. 2020;46(6):1294-1297.

10. Tavazzi G, Pellegrini C, Maurelli M, Belliato M, Sciutti F, Bottazzi A, Sepe PA, et al. Myocardial localization of coronavirus in COVID-19 cardiogenic shock. Eur J Heart Fail. 2020;22(5):911-915.

11. Cdc Covid-Response Team. Coronavirus Disease 2019 in Children - United States, February 12-April 2, 2020. MMWR Morb Mortal Wkly Rep. 2020;69(14):422-426.

12. Parri N, Lenge M, Buonsenso D, Coronavirus Infection in Pediatric Emergency Departments Research Group. Children with COVID-19 in pediatric emergency departments in Italy. N Engl J Med. 2020.

13. Barbaro RP, Paden ML, Guner YS, Raman L, Ryerson LM, Alexander P, Nasr VG, et al. Pediatric Extracorporeal Life Support Organization Registry International Report 2016. ASAIO J. 2017;63(4):456-463.

14. Steardo L, Steardo L, Jr., Zorec R, Verkhratsky A. Neuroinfection may contribute to pathophysiology and clinical manifestations of COVID-19. Acta Physiol (Oxf). 2020;229(3):e13473.

15. Troyer EA, Kohn JN, Hong S. Are we facing a crashing wave of neuropsychiatric sequelae of COVID-19? Neuropsychiatric symptoms and potential immunologic mechanisms. Brain Behav Immun. 2020. 